\title{
PSYCH up2date
}

\section{$5 \cdot 2017$}

\section{Neurotische, somatoforme und Belastungsstörungen 5}

\section{Zwangsstörungen im Kindes- und Jugendalter}

\author{
Susanne Walitza \\ Christa Rütter \\ Veronika Brezinka
}




\section{Unter dieser Rubrik sind bereits erschienen:}

Dermatillomanie (Skin-Picking-Störung): Diagnostik, Erklärung und Behandlung L. M. Mehrmann, A. L. Gerlach, A. Hunger Heft 4/2017

Spezifische Phobien A. Hamm Heft 3/2017

Diagnostik somatoformer Störungen C. Lahmann, C. Allwang, A. Dinkel Heft 5/2016

EMDR in der Psychotherapie: Einsatzmöglichkeiten, Wirksamkeit und Begrenzungen M. Stephan Heft 4/2016

Panikstörung und Agoraphobie P. Kindermann, M. Mühlberger, U. Voderholzer Heft 2/2016

Pharmakotherapie bei Angsterkrankungen P. Zwanzger Heft 2/2016

Die körperdysmorphe Störung: aktuelle Entwicklungen zu Diagnostik, Störungswissen und Therapie K. Schieber, A. Martin Heft $1 / 2016$

Soziale Angststörung J. Lin, I. Struina, U. Stangier Heft 2/2014

Störungsspezifische Therapie der Zwangsstörungen

U. Voderholzer, M. Müller, A. Külz Heft 1/2014

Angststörungen im Kindes- und Jugendalter S. Schneider, S. Seehagen Heft 6/2013

Behandlung der posttraumatischen Belastungsstörung

R. Rosner, A. Nocon, M. Olff Heft 5/2013

Generalisierte Angststörung J. Hoyer, J. Plag Heft 2/2013
Pathologisches Horten und Sammeln als Erkrankung des Zwangsspektrums D. Schön, A. Wahl-Kordon, B. Zurowski Heft $1 / 2013$

Das Depersonalisations-Derealisationssyndrom M. Michal Heft 1/2013

Emotionsbezogene Techniken in der Psychotherapie G. Jacob, C.-H. Lammers Heft 5/2012

Zwangsspektrumerkrankungen M. Anlauf, A. Kordon Heft 3/2010

Anpassungsstörungen S. Elstner, A. Diefenbacher Heft 1/2009

Dissoziative Störungen K. Priebe, C. Schmahl Heft 4/2008

Somatoforme Störungen und Hypochondrie R. Mewes, W. Rief Heft 3/2008

Panikstörung und Agoraphobie N. Vriends, J. Margraf Heft 2/2008

Diagnostik der Generalisierten Angststörungen T. Bär, M. Linden Heft $1 / 2008$

Soziale Phobien erkennen und behandeln K. Consbruch, U. Stangier Heft 4/2007

Zwangsstörungen U. Voderholzer, A. Külz Heft 3/2007

Posttraumatische Belastungsstörungen U. Schnyder Heft $1 / 2007$

\section{ALLES ONLINE LESEN}

Mit der eRef lesen Sie Ihre Zeitschrift: online wie offline, am PC und mobil, alle bereits erschienenen Artikel. Für Abonnenten kostenlos! https://eref.thieme.de/psych-u2d

\section{JETZT FREISCHALTEN}

Sie haben Ihre Zeitschrift noch nicht freigeschaltet? Ein Klick genügt: www.thieme.de/eref-registrierung 


\section{Zwangsstörungen im Kindes- und Jugendalter}

Susanne Walitza, Christa Rütter, Veronika Brezinka

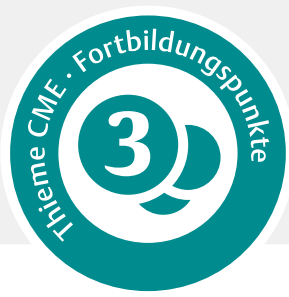

\section{Zwangsstörungen sind für viele Kinder und Jugendliche sehr beeinträchtigende Störungen. Zwangshandlungen und Zwangsgedanken treten häufig gemeinsam auf. Mangelnde Einsichtsfähigkeit, komorbide Störungen und die Interaktionen in der Familie müssen bei der Diagnostik und Behandlung besonders berücksichtigt werden.}

\section{Einleitung}

Zwangsstörungen sind auch schon im Kindes- und Jugendalter häufig auftretende und sehr beeinträchtigende Erkrankungen. Erwachsene mit Zwangsstörungen leiden häufig bereits seit ihrer Kindheit an Zwängen. Die Erkrankung wird oftmals lange verheimlicht oder bei Kindern nicht richtig erkannt. Dies führt dazu, dass sich trotz hoher Prävalenz nur ein Bruchteil der Betroffenen in Therapie befindet oder rechtzeitig eine Behandlung begonnen hat. Ein anderes Dilemma ist, dass sich immer noch nur relativ wenige Therapeuten auf eine - speziell zu Beginn - sehr aufwendige Therapie mit Expositionen und Reaktionsverhinderung einlassen. Sei es, da sie sich eine solche Behandlung nicht zutrauen oder immer noch Skrupel vor Expositionen haben. Auch ökonomisch lohnen sich diese zu Beginn der Expositionen oft langen Sitzungen eventuell nicht oder sind, wenn sie zu Hause stattfinden sollten, schwer zu organisieren. Viele verschiedene Gründe führen dazu, dass eine Vielzahl von Patienten keine evidenzbasierte Behandlung erhält. Der Beitrag soll das Erkennen und Verstehen von Zwangsstörungen im Kindes- und Jugendalter verbessern und die Hemmschwelle erniedrigen, diese Patienten zu behandeln.

\section{Symptomatik und Klassifikation}

\section{Symptomatik}

Zwangsstörungen sind komplexe Störungsbilder mit vielfältigen Erscheinungsformen. Die wichtigsten Symptomkomplexe sind:

- Zwangshandlungen und

- Zwangsgedanken.

\section{Merke}

Zwangshandlungen und Zwangsgedanken können unabhängig voneinander vorkommen, sie treten jedoch häufig gemeinsam auf - dann als „gemischt“ klassifiziert.

\section{Zwangsgedanken}

Bei Zwangsgedanken handelt es sich um Vorstellungen oder Ideen, die sich wiederholt und gegen den Willen des Betroffenen aufdrängen, als unsinnig erlebt werden und schweres Unbehagen auslösen. Die als unangenehm empfundenen Zwangsgedanken sind meist mit dem Drang verbunden, sie zu beenden oder ungeschehen zu machen - sie sozusagen zu neutralisieren.

Inhaltlich beziehen sich Zwangsgedanken häufig auf Angst vor Kontaminationen und drohenden Gefahren (Schmutz, Krankheitserreger), aber auch auf Aggression, Symmetrie und Genauigkeit.

\section{Merke}

Die „Ausgestaltung“ der Zwangsinhalte ist oft abhängig vom Alter und der Entwicklungsphase bzw. den Entwicklungsaufgaben des Betroffenen.

Während junge Kinder vermehrt Zwänge beim Anziehen oder Toilettengang zeigen, werden im Jugendalter religiöse oder sexuelle Themen sowie das Bedürfnis nach Autonomie bedeutsamer. Gedanken, man könnte mit jedem Toilettengang eine Schwangerschaft verursachen oder man könnte die eigenen Eltern töten wollen, belasten die Betroffenen extrem und können starke Schamgefühle auslösen. Mischformen und Kombinationen verschiedener Zwangsinhalte kommen sehr häufig vor. Ebenfalls ist die Persistenz der Zwangsinhalte im Kindesund Jugendalter geringer als im Erwachsenenalter (Wechsel z. B. von Symmetrie- zu Kontrollzwängen). Die Symptome können auch episodisch auftreten. 


\section{DEFINITION}

\section{Zwangsgedanken nach ICD-10 (F42.0) [1]}

- Unter Zwangsgedanken versteht man zwanghafte Ideen, Gedanken, Vorstellungen oder Impulse mit wiederholtem Auftreten.

- Sie werden von den Betroffenen meist als quälend, störend oder ungewollt empfunden, weshalb sie versuchen, den Gedanken Widerstand zu leisten.

- Die Ideen können unterschiedliche Inhalte haben und sind häufig sinnlos.

\section{Zwangshandlungen}

Unter Zwangshandlungen versteht man Verhaltensweisen, die einer bestimmten Regelhaftigkeit folgen, um Angst, eine drohende Gefahr oder ein Unbehagen zu reduzieren oder zu vermeiden. Die Einsicht in die Unsinnigkeit dieser Verhaltensweisen kann bei den Betroffenen graduell sehr verschieden sein. Bei Kindern können sowohl die Einsicht der Unsinnigkeit als auch der Widerstand gegen die Symptomatik gänzlich fehlen. Die häufigsten Zwangshandlungen im Kinder- und Jugendalter sind Reinigungszwänge - wie Wasch- und Putzzwänge oder auch zwanghaftes Kontrollieren. Es lassen sich aber auch sehr bizarr anmutende Zwangshandlungen beobachten, z. B. Luft verwirbeln, um eigene Schuppen der Haare aufzufangen oder bestimmte Formen in der Wohnung „100-mal“ entlanglaufen, um Schlimmes zu verhindern. Diese Formen der Zwangshandlungen müssen teilweise von psychotischen Störungen abgegrenzt werden.

\section{DEFINITION}

\section{Zwangshandlungen nach ICD-10 (F42.1) [1]}

- Zwangshandlungen sind wiederholte Verhaltensweisen, die einer gewissen Regelhaftigkeit folgen und denen die Furcht vor einer bedrohlichen Situation zugrunde liegt. Das Verhalten ist ein meist wirkungsloser Versuch, diese potenziell gefährliche Situation zu vermeiden.

- Häufig beziehen sich die Handlungen auf die Reinlichkeit, übertriebene Ordnung und Sauberkeit sowie wiederholtes Kontrollieren.

- Zwangshandlungen kommen bei beiden Geschlechtern gleich häufig vor, wobei sich das spezifisch gezeigte Verhalten unterscheidet.

- Im Vergleich zu Zwangsgedanken sind Zwangshandlungen weniger eng mit Depression verbunden und schlagen besser auf eine Verhaltenstherapie an.

\section{Zwangsimpulse}

Im DSM-5 wird nicht mehr von Zwangsimpulsen gesprochen, sondern vom „Drang/Sich-Aufdrängen“ (engl.: Urge) gewisser Gedanken oder Verhaltensweisen. Im Gegensatz zu Patienten mit Impulskontrollstörungen im engeren Sinn gibt der Patient mit Zwangsstörung diesen Impulsen, nun Drang genannt, in der Regel nicht nach. Während Patienten mit einer Impulskontrollstörung (z.B. ADHS) erst nach dem Ausführen des Impulses über ihre Handlung nachdenken, wird bei einer Zwangsstörung die Ausführung des Impulses gefürchtet und spezifische Situationen werden vermieden oder neutralisiert, damit es nie dazu kommen kann. Solche sehr angstauslösenden Drangvorstellungen (z. B. ich könnte ein Messer aus der Schublade nehmen und jemanden damit erstechen) können sich mit großer Macht aufdrängen und werden dann in der Regel mit sehr zeit- und kraftraubenden „Gegenmaßnahmen “ verhindert. So wird beispielsweise die Küchentür und somit der Zugang zu den Messern x-mal abgeschlossen und kontrolliert. Diese Impulse können den Betroffenen so ängstigen, dass er aus Sorge, er könnte schlafwandeln und sich die Messer holen, nicht mehr schlafen kann.

\section{Klassifikation}

Die Klassifikation erfolgt in den deutschsprachigen Ländern in der Regel nach der ICD-10.

\section{Merke}

Bei Zwangsstörungen im Kindes- und Jugendalter empfiehlt sich alternativ oder mindestens zusätzlich zum ICD-10 eine Betrachtung der DSM-5 Kriterien.

Das DSM-5 berücksichtigt bei Minderjährigen die noch teilweise mangelnde Fähigkeit, die Zwangssymptome als eigene Gedanken zu erkennen und ihnen Widerstand zu leisten. Entscheidend ist bei beiden Klassifikationsschemata, dass die Zwangssymptome auch schon bei Kindern, die noch wenig Einsicht zeigen können, einen hohen Leidensdruck auslösen. Zudem wird im Gegensatz zu Ritualen die Wiederholung der Zwangshandlungen als belastend erlebt.

Das DSM-5 hat einige Neuerungen gegenüber dem ICD10 sowie Anpassungen gegenüber dem DSM-IV eingeführt [3]. Nach DSM-IV waren Zwangsstörungen unter der Kategorie der „Angststörungen“ eingeordnet. Im DSM-5 gibt es nun eine eigene Kategorie für „Zwangsstörungen und verwandte Störungen“. Gemeinsam ist den Angst- und Zwangsstörungen oftmals eine zugrunde liegende, tiefe Angst, deren Auslöser oder potenzielle Auslöser bei der Angststörung vor allem vermieden werden und bei der Zwangsstörung z. B. durch Zwangshandlungen neutralisiert werden. Es gibt aber auch Zwänge, denen nicht per se eine Angst zugrunde liegt (z. B. Symmetriezwänge oder „Just-right“-Zwänge). Hier entsteht erst durch das Unterlassen dieser Zwänge ein sehr unangenehmes, z. B. „unfertiges“ Gefühl. 
Die neue Kategorie der Zwangsstörungen im DSM-5 setzt sich aus einem Zwangsspektrum von Zwangsstörungen im engeren Sinne (s.o.) mit verschiedenen Störungsbildern zusammen.

\section{ZUSATZINFO}

\section{Den Zwangsstörungen verwandte Störungen nach DSM-5 [2]}

- Dysmorphophobie/Body Dysmorphic Disorder

- Horten

- Trichotillomanie

- Exkoriationsstörung (Skin Picking)

- durch Substanz oder Medikation induzierte Zwangsstörung

- Zwänge oder verwandte Störungen bedingt durch eine andere medizinische Ursache

- andere spezifische Zwangsstörungen und verwandte Störungen

- unspezifische Zwangsstörungen und verwandte Störungen

\section{Bedeutung der Einsichtsfähigkeit}

Neu und auch therapeutisch relevant sind die Spezifizierungen des DSM-5 zur Einschätzung der Einsichtsfähigkeit. Die Einsichtsfähigkeit muss nun als „gut/angemessen“, „eingeschränkt“ oder „fehlend“ eingeschätzt werden. Dieses Kriterium ist auch von besonderer Bedeutung zur Abgrenzung von Psychosen, bei denen „ich-syntone“ zwangsartige Vorstellungen oder Ideen auftreten können, also auch „keine Einsicht“, aber in der Regel liegen bei diesen Patienten auch andere Symptome einer psychotischen Störung vor [3]. Körperdysmorphe Störungen ohne Einsicht werden nur noch unter der Gruppe der Zwangsstörungen und nicht mehr bei den psychotischen Störungen klassifiziert, selbst wenn diese wahnhafte Formen annehmen [4]. In den wenigen vorliegenden Untersuchungen bei Kindern und Jugendlichen, konnte gezeigt werden, dass die Einsichtsfähigkeit negativ mit dem Alter korreliert und auch bei einem höheren Schweregrad der Zwänge und bei gleichzeitig vorliegenden Angststörungen reduziert ist $[5,6]$. Bei reduzierter Einsichtsfähigkeit wurde eine schlechtere Therapieresponse auf SSRIs, aber auch auf verhaltenstherapeutische Interventionen beobachtet [7].

\section{TIPP FÜR DIE PRAXIS}

Beurteilen Sie immer die Einsichtsfähigkeit der Betroffenen. Bei Kindern ist diese oft allein aufgrund des Entwicklungsstandes reduziert. Trotzdem muss bei fehlender oder eingeschränkter Einsichtsfähigkeit immer die Differenzialdiagnose einer Psychose bedacht werden.

\section{DIAGNOSEKRITERIEN}

\section{Zwangsstörungen nach ICD-10 (F42) [1]}

A. Zwangsgedanken oder Zwangshandlungen (oder beides) sind an den meisten Tagen über einen Zeitraum von mindestens 2 Wochen nachweisbar.

B. Die Zwangsgedanken/-handlungen müssen sämtliche folgende Merkmale aufweisen:

- Sie werden vom Betroffenen als eigene Gedanken oder Handlungen angesehen und werden nicht als von anderen Personen eingegeben empfunden.

- Ihr wiederholtes Auftreten wird vom Betroffenen als unangenehm empfunden und als unsinnig oder übertrieben angesehen.

- Gegen mindestens einen dieser Gedanken oder Handlungen wird Widerstand geleistet, auch wenn wenig erfolgreich.

- Sie werden vom Betroffenen nicht als angenehm empfunden, wobei von einer vorübergehenden Erleichterung von einem angespannten Zustand unterschieden werden muss.

C. Die Zwangsgedanken und -handlungen lösen einen Leidensdruck aus und beeinträchtigen die soziale oder individuelle Leistungsfähigkeit.

D.Die Störung tritt nicht aufgrund einer anderen psychischen Störung wie Schizophrenie oder affektive Störungen auf.

\section{Zwangsstörungen nach dem DSM-5 [2]}

A. Vorhandensein von Zwangsgedanken und/oder Zwangshandlungen

Zwangsgedanken sind definiert durch:

- Wiederkehrende, stabile Gedanken, Vorstellungen oder Ideen, die vom Betroffenen als unangenehm empfunden werden und einen Leidensdruck hervorrufen.

- Die Betroffenen versuchen, diese Gedanken zu unterdrücken, zu ignorieren oder ihnen mit einem anderen Gedanken oder einer Handlung entgegenzuwirken.

Zwangshandlungen sind definiert durch:

- Wiederkehrende Verhaltensweisen oder vorgestellte Handlungen, welche vom Betroffenen in einer gewissen Regelhaftigkeit ausgeführt werden.

- Diese Verhaltensweisen oder vorgestellten Handlungen haben das Ziel, die Angst vor einer bedrohlichen befürchteten Situationen zu reduzieren. Da diese Handlungen jedoch in keiner realistischen Weise mit diesem Ziel verbunden sind, bleibt dies erfolglos.

B. Die Zwangsgedanken und -handlungen führen über den hohen Zeitaufwand zu einem klinisch relevanten Leidensdruck oder zu einer Beeinträchtigung sozial oder individuell bedeutsamer Lebensbereiche.

C. Den Zwangssymptomen liegen keine physiologischen Effekte einer Substanz oder eines Medikaments zugrunde.

D.Die Symptome können nicht besser durch eine andere psychische Störung erklärt werden (z. B. übermäßige Sorgen bei einer generalisierten Angststörung). 


\section{Komorbiditäten}

Komorbide psychische Störungen sind wie bei anderen psychischen Störungen im Kindes- und Jugendalter sehr verbreitet und liegen bei Zwangsstörungen mit bis zu $70 \%$ vor $[3,8,9]$.

\section{Merke}

Je ausgeprägter die Zwangserkrankung, umso wahrscheinlicher scheinen auch Komorbiditäten aufzutreten [10].

Die relevantesten Komorbiditäten der Zwangsstörung im Kindes- und Jugendalter sind unter $>$ Tab. 1 gelistet.

- Tab. 1 Komorbidität der Zwangsstörungen über das gesamte Kindes- und Jugendalter (Mediane) nach Wewetzer [11].

\begin{tabular}{|l|l|}
\hline Komorbidität & Häufigkeit \\
\hline Angststörungen & $38 \%$ \\
\hline Depressionen & $33 \%$ \\
\hline ADHS und expansive Störungen & $29 \%$ \\
\hline Tic-Störungen & $20 \%$ \\
\hline
\end{tabular}

Bei Knaben und jüngeren Kindern sind Aufmerksamkeitsdefizit- und Hyperaktivitätsstörung (ADHS) und Tic-Störungen hervorzuheben, wohingegen bei Mädchen und Jugendlichen eher Essstörungen zusätzlich auftreten. Angststörungen sowie depressive Störungen treten bei allen Altersgruppen und geschlechtsunabhängig häufig komorbid auf. Mit zunehmendem Alter können auch Persönlichkeitsstörungen hinzukommen.

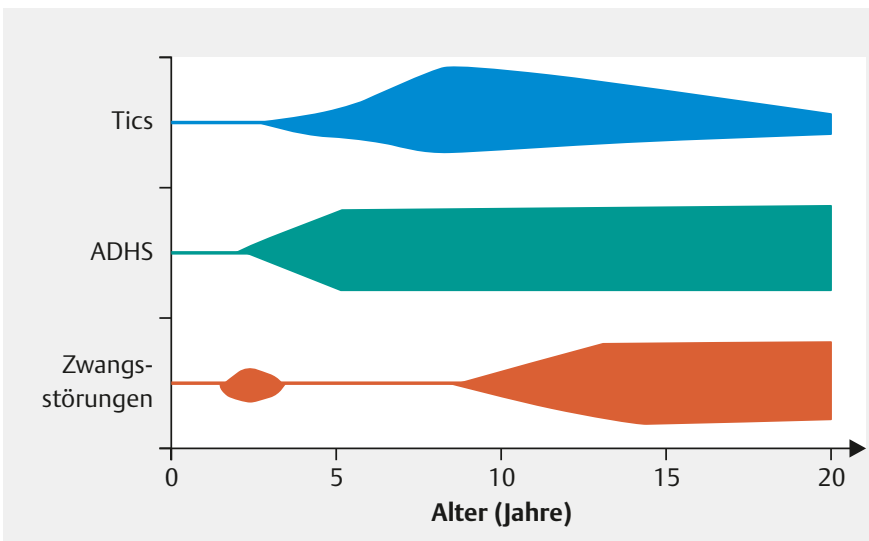

- Abb. 1 Altersabhängiger Beginn und Verlauf von Tics, ADHS und Zwang (nach [15]).
Das DSM-5 verlangt ebenfalls neu im Vergleich zum DSM-IV ausdrücklich eine Spezifizierung eines Subtyps von Zwangsstörungen mit einer zusätzlich auftretenden Tic-Störung. Diskutiert werden gemeinsame genetische Risikofaktoren für Zwänge und Tics, aber auch für ADHS, da z. B. diesen Störungen eine Inhibitionsstörung zugrunde zu liegen scheint. Auch scheint es eine eher motorische Form von Zwängen (ohne primär zugrunde liegender Angst) zu geben, die den Tic-Störungen verwandt zu sein scheint (siehe auch [12]). Die komorbiden Störungen wurden auch als Prädiktoren für den Behandlungserfolg beschrieben. Patienten mit Zwängen und gleichzeitigen Tic-Störungen, ADHS oder einer Störung mit oppositionellem Trotzverhalten sprachen weniger positiv auf SSRI und auch auf kognitiv-verhaltenstherapeutische Behandlung an $[13,14]$.

\section{TIPP FÜR DIE PRAXIS}

Im DMS-5 muss neu immer die Einsichtsfähigkeit und die Komorbidität mit Tic-Störungen beurteilt werden. Beide Symptombereiche sind wesentliche Prädiktoren für das Ansprechen auf die Behandlung.

Der potenziell gemeinsame Verlauf der häufig auftretenden, komorbiden Störungen (nach [15]) zeigt, dass ADHS und Tic-Störungen ihren Erkrankungsbeginn immer im Kindesalter haben und ein Teil der Zwangsstörungen ebenfalls in einer ähnlichen Altersgruppe und teilweise sogar besonders früh beginnt. Im Vergleich zu Zwängen und ADHS reduzieren sich die Tic-Störungen tendenziell mit der Zeit deutlicher von selbst ( $\bullet$ Abb. 1; siehe auch [12]).

Differenzialdiagnostisch sind Zwangshandlungen teilweise schwer von komplexen Tic-Störungen abzugrenzen. Vor allem, wenn komplexe Tics ein ganzes Handlungsmuster umfassen, wie z.B. Hüpfen und Springen oder Klopfen oder mit den Fingern speziell tippen etc. Entscheidend ist aber in der Regel, dass hinter dem Zwang eine Intention zu finden ist, was bei Tic-Störungen so nicht der Fall ist.

\footnotetext{
Merke

Die Zwangshandlung ist für den Patienten mit Zwangsstörung nicht per se angenehm, sondern durch die ständige Wiederholung sehr quälend. Die sich dauernd wiederholenden Tics hingegen sind meistens eher für die Umwelt unangenehm. Die Patienten leiden oft mehr unter der Reaktion der Umwelt als unter dem Tic selbst.
} 
TIPP FÜR DIE PRAXIS

Für differenzialdiagnostische Beurteilung von Zwängen und Tics sollte man den Zweck, der dem Verhalten zugrunde liegt, betrachten. Während der Zwang oftmals einen Zweck verfolgt (z. B. eine Angst zu reduzieren), dient ein sich wiederholender Tic keinem Zweck.

\section{Epidemiologie und Prävalenz}

Zwangsstörungen treten kulturübergreifend in allen Teilen der Welt auf. Die Prävalenz im Kindes- und Jugendalter beträgt etwa 1-3\% [3]. Nach der „Comorbidity Survey Replication“ war etwa jeder fünfte Betroffene bereits vor dem Alter von 10 Jahren an seiner Zwangsstörung erkrankt [16]. Die Altersverteilung ist bimodal, ein erster Erkrankungsgipfel scheint in der Kindheit im 11. Lebensjahr und ein zweites Maximum im frühen Erwachsenenalter im 23. Lebensjahr vorzuliegen [17]. Es gibt Hinweise darauf, dass im Kindesalter Jungen etwas häufiger betroffen sind als Mädchen. Ab dem Jugendalter wurden jedoch keine bedeutsamen Geschlechtsunterschiede mehr beschrieben.

\section{Verlauf}

Verlaufsstudien im Kindes- und Jugendalter zeigen wie im Erwachsenenalter eine deutliche Chronifizierung. In einer Metaanalyse und in einer deutschsprachigen prospektiven Verlaufsstudie wurden 5 Jahre nach Beginn der Erkrankung mittlere Persistenzraten von $40 \%$ für das Vollbild und von $60 \%$ unter Berücksichtigung subklinisch ausgeprägter Zwangsstörungen beobachtet [18]. Untersuchungen zum Schweregrad direkt nach der Therapie sowie zum Follow-up-Zeitpunkt zeigen jedoch jeweils signifikante Verbesserungen [19-21]. Zusammenfassend scheinen viele Patienten auch noch nach einer Therapie die Kriterien der Zwangsstörung zu erfüllen, wobei aber der Schweregrad deutlich abgenommen hat und die Beeinträchtigung soweit reduziert war, dass die Betroffenen eine gute Lebensqualität erleben. Für einen guten oder negativen Verlauf scheinen besonders relevant die soziale Integration, die altersgemäße Verselbstständigung von der Herkunftsfamilie und der Partnerschaft [19]. Prognostisch günstig erwiesen sich vor allem

- ein früher Behandlungsbeginn und

- die Fortsetzung einer begonnenen Therapie [18].

Der Früherkennung einer Zwangsstörung kommt daher eine besondere Bedeutung zu, da sie die Prognose entscheidend verbessern kann.
Merke

Je früher Zwangsstörungen erkannt werden, umso besser ist die Prognose. Je früher die Behandlung beginnt, umso besser ist die psychosoziale Entwicklung im Verlauf der Zwangsstörung.

TIPP FÜR DIE PRAXIS

Bei Verdacht auf eine Zwangsstörung im Kindes- und Jugendalter warten Sie nicht lange, sondern konsultieren Sie bald einen kinder- und jugendpsychiatrischen Experten.

\section{Ursachen und aufrechterhaltende Faktoren}

Neben psychosozialen Ursachen, die Auslöser für Zwangsstörungen und bedeutsame aufrechterhaltende Faktoren sein können, sind auch biologische Faktoren für die Entstehung von Zwangsstörungen relevant. Insbesondere bei Patienten mit sehr frühem Erkrankungsbeginn stellt sich die Frage einer biologischen Vulnerabilität, wobei aber dem „Modell-Lernen“ in diesem Alter eine ebenso wichtige Rolle zukommt. Für die Bedeutung beider Faktoren gibt es viel Evidenz und beide Faktoren sind letztlich für die Behandlung relevant.

\section{Genetische Komponenten}

Zwillingsstudien zeigen, dass die Konkordanz bei monozygoten Zwillingen bei einem frühen Erkrankungsbeginn höher war als bei Patienten mit Erkrankungsbeginn im Erwachsenenalter [22]. In einer aktuellen Studie wurden alle dänischen Psychiatriepatienten mit einer Zwangsstörung der Geburtsjahrgänge 1952-2000 untersucht. Es zeigte sich klar, dass in der Patientengruppe signifikant mehr Familienmitglieder ebenfalls unter Zwängen litten. Zusammenhänge mit dem Erkrankungsbeginn wurden jedoch nicht gefunden. Neben der familiären Häufung von Zwangskrankheiten waren auch Tic-Störungen, Affektstörungen sowie Angststörungen bei den Verwandten, ein mütterliches Geburtsalter von über 35 Jahren, ein jüngeres Alter der Patienten sowie tendenziell auch das männliche Geschlecht Risikofaktoren für die Entwicklung von Zwangsstörungen [23].

Neben einer großen Gruppe der sogenannten eher „familiär" auftretenden Zwangsstörungen findet man aber nahezu genauso häufig sog. „neu erkrankte Patienten, ohne familiäre Belastung“. In den meisten bisherigen molekulargenetischen Studien ist es noch nicht gelungen, einzelne Gene, Genvarianten oder in den neuesten Untersuchungen z. B. CNV (Copy Number Variations) mit hohen Effektstärken als Ursache für Zwangsstörungen zu finden. Die bisher vielversprechendsten Befunde umfassen Gene in serotonergen, dopaminergen und gluta- 


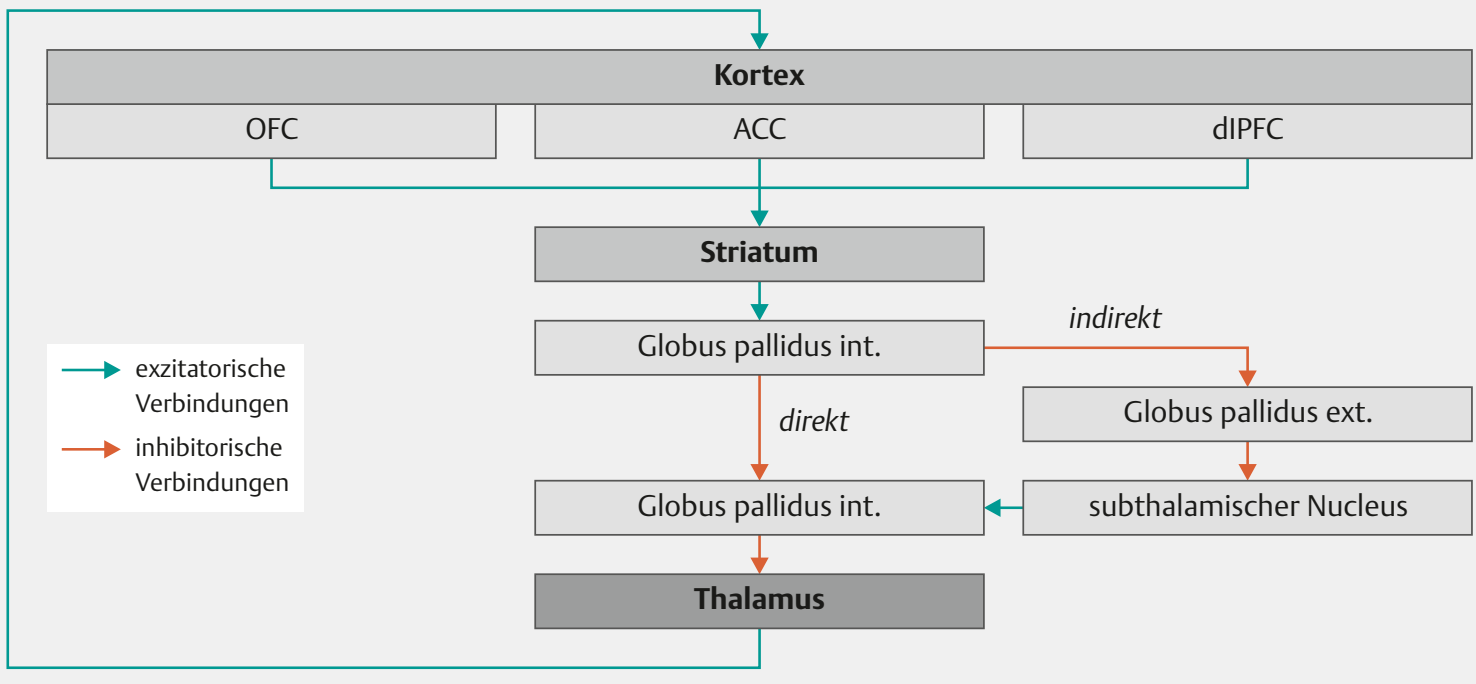

- Abb. 2 Vereinfachte Darstellung der kortiko-striato-thalamischen (CST) Bahnen (nach [26]). Abkürzungen: OFC= orbitofrontaler Kortex, ACC = anteriorer cingulärer Kortex, dIPFC = dorsolateraler präfrontaler Kortex.

matergen Systemen [24]. Diese Gene haben auch für andere psychische Störungen eine Relevanz, wobei aktuell noch unklar ist, welche zusätzlichen Faktoren, u. a. epigenetische Faktoren, für die Entstehung der jeweiligen psychischen Störungen ausschlaggebend sind.

\section{Neurobiologische Befunde}

Grundsätzlich geht man davon aus, dass bei Patienten mit Zwängen sog. kortico-striato-thalamische (CST) Regelkreisläufe beeinträchtigt sind ( $\bullet$ Abb. 2) [13]. Diese Netzwerke sind wichtige Feedbackschlaufen für motorische, kognitive und affektive Prozesse; sie regulieren die Aktivität in den involvierten Hirnregionen. Bei Patienten mit Zwangsstörungen scheinen sich diese Kreisläufe jedoch nicht im Gleichgewicht zu befinden, was sich durch strukturelle Bildgebungsbefunde und Aktivierungsunterschiede in den beteiligten Regionen zeigt [25]. Funktionelle Studien zeigen Auffälligkeiten bei Lern- und Inhibitionsaufgaben. Nach einer Metaanalyse von Brem und Kollegen findet sich eine Hypoaktivierung insbesondere im präfrontalen Kortex und im anterioren Cingulum (ACC) - einer Region, die auch mit immer wiederkehrenden Gedanken in Verbindung gebracht wird [26].

Die Resultate der funktionellen Bildgebung deuten zusammenfassend auf Aktivierungsunterschiede bei Zwangspatienten in den entsprechenden funktionellen Gehirnnetzwerken hin [26, 27]. Ein Ungleichgewicht der direkten und indirekten Pfade der CST-Kreisläufe könnte die Hyper- oder Hypoaktivität von spezifischen Gehirnarealen verursachen, die in funktionalen Bildgebungsstudien genannt werden. Zudem können die Symptome von Zwangspatienten möglicherweise auch durch ein Un- gleichgewicht in den parallelen kognitiven und affektiven Kreisläufen erklärt werden.

\section{Immunologische Faktoren}

Zwangssymptome (häufiger auch Tic-Störungen), die in engem Zusammenhang mit Infektionen durch beta-hämolysierende Streptokokken auftreten, werden unter der Gruppe PANDAS (Pediatric autoimmune neuropsychiatric Disorders associated with streptococcal Infections) beschrieben. Die Ursache scheint eine Kreuzreaktion zu sein: von primär gegen beta-hämolysierende Streptokokken der Gruppe A gerichteten Antikörpern gegen Basalganglien. Die Prävalenz ist noch unbekannt.

Die Symptome beginnen im Kindesalter (meist zwischen 3 und 12 Jahren) und treten abrupt und oft mit dramatischer Symptomverschlechterung auf. Zwischen den verschiedenen Episoden bilden sich die Symptome jeweils wieder zurück, wobei Symptombeginn oder -zunahme in engem zeitlichen Zusammenhang mit dem Streptokokkeninfekt steht.

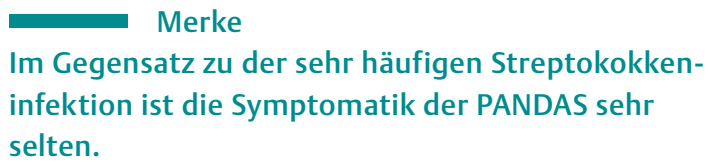

Snider und Kollegen zeigten erstmals die Wirksamkeit einer antibiotischen Prophylaxe [28]. PANDAS haben bislang weder im ICD-10 noch im DSM-5 Einzug gehalten und die Antibiotikaprophylaxe ist bislang mehr Theorie als Praxis. Das neuere Konstrukt sind die PANS (Pediatric acute-onset neuropsychiatric Syndrome) - Erkrankungen, denen der ebenfalls akute Beginn gemeinsam ist 
[29]. Die Symptomatik ist breiter als bei PANDAS und schließt neben Zwangsstörungen Störungen der Nahrungsaufnahme, aber auch Irritabilität und Depression mit ein. Die Pathogenese ist noch unklar, man geht aber von einem direkten Zusammenhang mit einer vorangegangenen oder aktuellen Infektion, metabolischen Störungen oder einer anderen Entzündungsreaktion aus.

\section{HINTERGRUNDWISSEN}

\section{Diagnostische Kriterien für PANS [29]}

A. Abrupter Beginn von deutlichen Zwangssymptomen oder der verminderten Nahrungsaufnahme

B. Mit gleichzeitigem Auftreten ähnlich akuter neurologischer Auffälligkeiten von mindestens 2 der folgenden Kategorien:

- Angst

- emotionale Instabilität oder Depression

- Aggressivität, Reizbarkeit oder oppositionelles Verhalten

- Rückschritt in der Verhaltensentwicklung

- Verschlechterung der schulischen Leistung

- sensorische oder motorische Auffälligkeiten

- somatische Symptome (Schlafprobleme, Enuresis oder Harndrang)

C. Die Symptome können durch keine anderen neurologischen oder medizinischen Störungsbilder erklärt werden.

\section{Kognitiv-behaviorale Modelle}

Entsprechend metakognitiv-behavioraler Befunde und Modelle liegen den Zwangsstörungen dysfunktionale Interpretationsmuster von wahrgenommenen Situationen, Ereignissen und Gedanken zugrunde ( $\mathbf{A b b}$. 3).

\section{Merke}

Die Zwangspatienten bewerten „eigentlich“ normale Gedanken als aufdringlich oder negativ.

Diese dysfunktionalen Interpretationsmuster führen unter anderem zu einer verstärkten Wahrnehmung von Gefahr und persönlicher Verantwortung. Dies resultiert in einem Drang, die als quälend erlebten Gedanken durch Vermeidungsverhalten zu beenden oder zu neutralisieren. Der Versuch, die aufdringlichen Gedanken nicht zu denken oder zu unterdrücken, hat keinen hilfreichen, sondern in der Regel einen gegenteiligen Effekt. Eine kurzfristig erlebte Erleichterung verstärkt das Verhalten, was die Bedeutung der Gedanken verstärkt. Dies führt zu einer weiteren Stabilisierung der ständigen Beschäftigung mit dem unangenehmen Gedanken. Das Unterdrücken verstärkt somit auf längere Sicht die Auseinandersetzung mit dem aufdringlichen Gedanken und hält die dysfunktionale Beurteilung aufrecht. Der Betroffene ist in einem Teufelskreis aus aufdringlichen Gedanken und Widerstandsversuchen gefangen.

Dieses Ursachenmodell von Salkovskis bildet die Grundlage für die kognitiv-behaviorale Therapie der Zwangsstörungen [30]. Die kognitiven Interventionen arbeiten an der Fehlinterpretation oder Überbewertung der Gedanken, wobei sich die verhaltenstherapeutischen Anteile

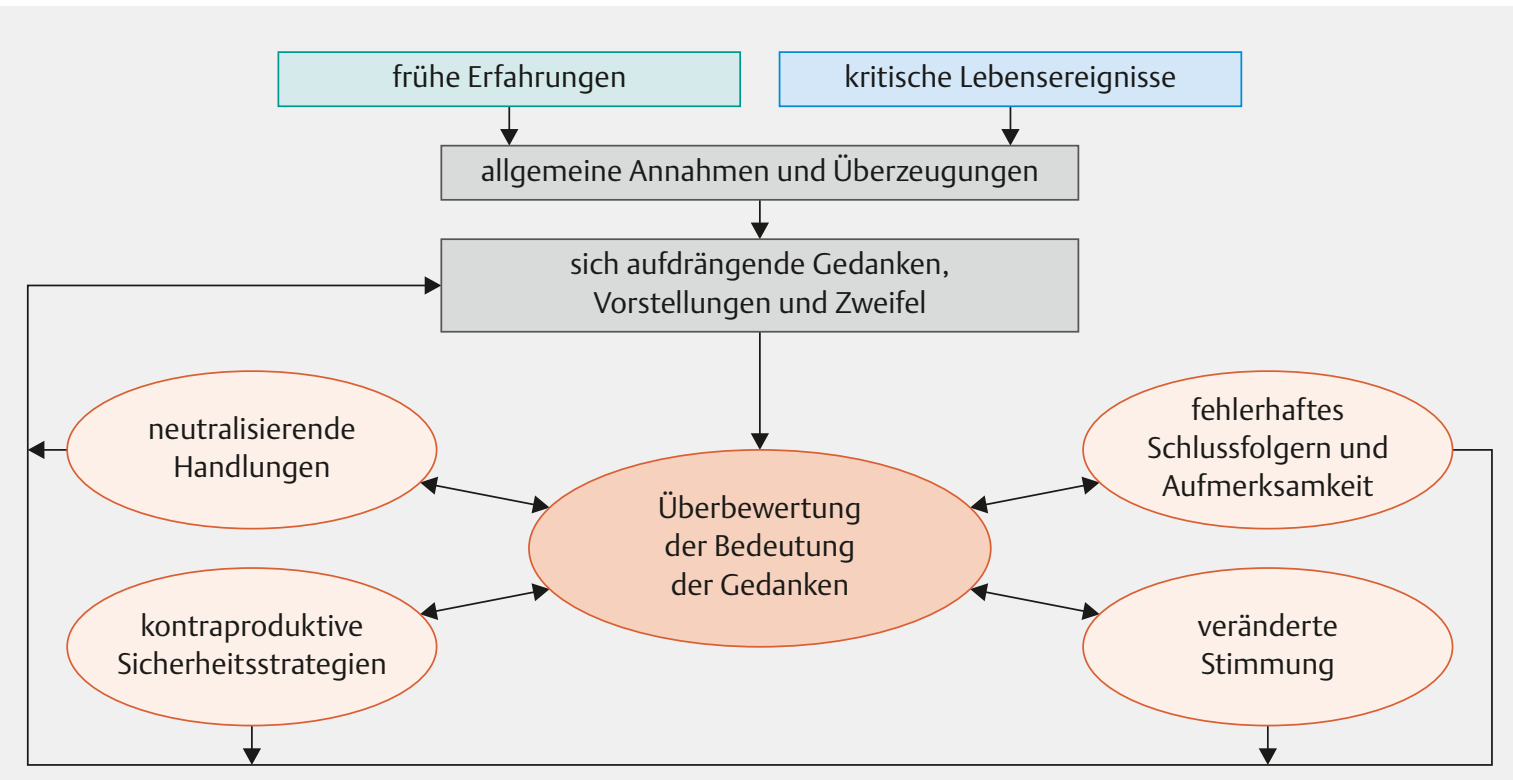

- Abb. 3 Modell der kognitiven Hypothese der Ursache von Zwängen (nach [30]). 
mit der Reaktionsverhinderung befassen (z. B. keine neutralisierenden Handlungen nach Exposition).

\section{TIPP FÜR DIE PRAXIS}

Das kognitive Modell ist bei Kindern und Jugendlichen nicht immer anwendbar, da die Kognitionen teilweise noch nicht ausreichend differenziert sind oder noch kaum Einsichtsfähigkeit vorliegt.

\section{Diagnostik von Zwangsstörungen im Kindes- und Jugendalter}

Die Diagnostik umfasst eine ausführliche Anamnese, Beobachtung, klinische und spezifische Untersuchung und Verhaltensanalyse.

\section{PRAXIS}

\section{Diagnostik: Verhaltensanalyse [31]}

- Erscheinungsbild und Schweregrad des Zwangs

- interne und externe Auslöser

- Erwartungen und Befürchtungen, falls die Zwangsrituale nicht ausgeführt werden könnten

- Möglichkeiten der Abwehr (mit welchen Verhaltensweisen kann sich der Patient bereits selbst behelfen?)

- Reaktionsweisen der (familiären) Bezugspersonen (Involvierung; protektive Ressourcen und verstärkende Einflüsse)

\section{Multiaxiales Klassifikationsschema}

Auf der ersten Achse wird das klinisch-psychiatrische Syndrom diagnostiziert (die Zwangserkrankung, aber auch komorbide psychische Störungen). Auf den weiteren Achsen werden umschriebene Entwicklungsstörungen beschrieben (Achse 2), das Intelligenzniveau betrachtet (Achse 3), eventuelle körperliche Symptome erfasst (Achse 4) sowie aktueller abnormer intrafamiliärer Beziehungen Rechnung getragen (Achse 5). Auf der 6. und letzten Achse erfolgt schließlich eine globale Beurteilung des psychosozialen Funktionsniveaus in mehreren Lebensbereichen. Die Ausprägung der Beeinträchtigung, die hier erfasst wird, ist mit der spezifischen Beurteilung des Schweregrads der Zwangsstörung entscheidend für die Intensität der Therapie.

Merke

Allgemein sollte die Diagnostik kinder- und jugendpsychiatrischer Störungen grundsätzlich in Anlehnung an das multiaxiale Klassifikationsschema für psychische Störungen des Kindes- und Jugendalters erfolgen [31].
Bei Verdacht auf eine Zwangsstörung sollte die gründliche kinder- und jugendpsychiatrische Abklärung (Achse 1) mit einem strukturierten klinischen Interview wie z.B. dem K-SADS [32] oder dem Kinder-DIPS erfolgen [33]. Zusätzlich sollte immer die Children's Yale-Brown Obsessive-Compulsive Scale CY-BOCS durchgeführt werden [34]. Eine Checkliste erfasst die einzelnen Symptomarten und im Interview werden verschiedene wesentliche Aspekte zum Schweregrad dezidiert erfasst (z. B. ob Widerstand geleistet werden kann). Ein Cut-off-Wert von 16 steht nach Scahill und Riddle für eine milde bis mittelschwere Zwangsstörung, während man ab einem Wert von 23 von einer mittelschweren bis schweren Symptomatik spricht [34].

Die CY-BOCS kann den Schweregrad unterschätzen, vor allem dann, wenn bei den Patienten keine Einsichtsfähigkeit vorliegt und sie nicht den Eindruck haben, dass die Zwänge sie beeinträchtigen oder z.B. viel Zeit kosten. Die Einsichtsfähigkeit selbst wird in der CY-BOCS aber miterfasst. Dies erlaubt in den meisten Fällen, die erhobene Einschätzung einzuordnen.

\section{TIPP FÜR DIE PRAXIS}

Das standardisierte Interview CY-BOCS gilt als internationaler Goldstandard für die Einschätzung des Schweregrades einer Zwangsstörung und sollte immer durchgeführt werden.

Die Einschätzung des Schweregrades der Erkrankung und die Diagnose von Komorbiditäten auf Achse 1 sind wesentlich, um die optimale Behandlung zu wählen sowie den Behandlungserfolg einzuschätzen [8].

\section{Behandlung}

Die erste Wahl der Behandlung von Zwangsstörungen im Kindes- und Jugendalter ist die kognitive Verhaltenstherapie. Dabei sind die Elemente der Konfrontation mit Reaktionsverhinderung zentral. Je jünger die Patienten sind, umso wesentlicher ist der Einbezug der Eltern und Familienmitglieder in die Therapie. Es gibt auch Indikationen für die Kombinationstherapie mit Medikation oder in sehr seltenen Fällen für die alleinige Therapie mit Psychopharmaka. Im Folgenden werden die einzelnen Therapieelemente vertieft.

\section{TIPP FÜR DIE PRAXIS}

Die Behandlung der ersten Wahl bei Zwangsstörungen im Kinder- und Jugendalter ist die kognitive Verhaltenstherapie. Eine frühe Behandlung über einen längeren Zeitraum unter Einbezug der Eltern und Familienmitglieder wird empfohlen. 
- Tab. 2 Drei Phasen der Therapie von Zwangsstörungen (nach [35]).

\begin{tabular}{|l|l|l|}
\hline Vorbereitung & Intensivphase & Nachsorge \\
\hline Pschoedukation & kognitive Interventionen & Rückfallprophylaxe \\
\hline individuelle Therapieplanung & Exposition & \\
\hline & (Pharmakotherapie) \\
\hline
\end{tabular}

Vor der eigentlichen Intervention steht die Diagnostik und Vorbereitung der Therapie, die sich in 3 Phasen einteilen lässt ( $>$ Tab. 2). Die Vorbereitungsphase folgt direkt der Diagnostik und schließt die Psychoedukation und Therapieplanung ein.

In der umfassenden Aufklärung über die Erkrankung können bereits Elemente der narrativen Therapie verwendet werden [36]. Im Sinne einer Metapher wird dabei „der Zwang" als ein Wesen (Tier, Krake, Monster etc.) externalisiert. Der Patient kann sich so in Gesprächen und Rollenspielen mit dem Zwang auseinandersetzen und sich von ihm abgrenzen. Gemeinsam mit Kind und Eltern wird ein Erklärungsmodell der Zwänge erarbeitet. Je nach Zwangssymptomatik und Entwicklungsstand werden dabei lerntheoretische, kognitive oder auch neurobiologische Aspekte sowie Persönlichkeit, familiäre und soziale Faktoren berücksichtigt.

\footnotetext{
Merke

Das kognitive Modell bietet sich z. B. immer dann an, wenn Zwangsgedanken sehr dominant sind, wohingegen das lerntheoretische Modell für die Erklärung von Zwangshandlungen gut geeignet ist.
}

Zu Verstehen und zu akzeptieren, dass es neurobiologische Ursachen von Zwängen gibt, entlastet meist bereits den Patienten und dessen Eltern, speziell, wenn die Erkrankung als eine Folge falscher Erziehung oder auch Schuld verstanden wird.

\section{TIPP FÜR DIE PRAXIS}

Das therapeutische Vorgehen sollte auf transparente und verständliche Art vermittelt werden, damit der Patient stets die Kontrolle über den Therapieprozess behält. Dieses Vorgehen fördert die Compliance und stärkt die Therapeut-Patient-Beziehung.

\section{Psychotherapie}

\section{Kognitive und Verhaltenstherapien}

Kognitive Therapieverfahren gehören zum Standard bei der Behandlung von Zwangsstörungen, da der Überbewertung von Gedanken ein entscheidender Faktor bei der Entstehung von Zwangsgedanken zukommt (kognitives Modell) [30,37]. Kognitive Interventionen unterstützen die direkte Auseinandersetzung mit den Zwangs- gedanken und -befürchtungen. Dem Patienten wird vermittelt, dass die Vermeidung und Unterdrückung schlimmer Gedanken nicht funktioniert, denn je mehr man versucht einen Gedanken nicht zu denken, desto häufiger und drängender tritt dieser auf („paradoxer Effekt der Gedankenunterdrückung“).

\begin{abstract}
Merke
Der Schwerpunkt kognitiver Interventionen liegt auf der Identifikation und Veränderung von dysfunktionalen Denkinhalten (kognitive Umstrukturierung).
\end{abstract}

Kognitive Interventionen können auch das Ziel haben, dysfunktionale Einstellungen zu verändern. Damit sind Einstellungen gemeint, die bei der Entstehung und Aufrechterhaltung der Zwänge relevant sind. Ein übertriebenes Verantwortungsgefühl, wie es z. B. auch in der CYBOCS erfragt wird, kann den „Nährboden“ für massive Ängste und Befürchtungen bilden.

\section{Exposition mit Reaktionsmanagement}

Die Exposition mit Reaktionsmanagement (ERM) ist ein zentrales Wirkelement der kognitiven Verhaltenstherapie. Bei der ERM konfrontiert sich der Patient mit den von ihm gefürchteten Situationen oder Gegenständen, ohne Zwangshandlungen auszuführen oder die Situation zu vermeiden. Die dadurch entstehenden negativen Gedanken, Gefühle (z. B. Angst, Sorge, Ekel etc.) oder körperlichen Symptome werden so lange ausgehalten, bis diese von selber in ihrer Intensität abnehmen. Das Prinzip der Gewöhnung (Habituation) führt dazu, dass die Reaktion eines Organismus auf den gleichen Reiz bei wiederholter Darbietung abnimmt.

\section{TIPP FÜR DIE PRAXIS}

Im Kinder- und Jugendbereich ist eine graduierte Expositionsbehandlung (ansteigender Schwierigkeitsgrad) grundsätzlich einem massierten Vorgehen (sofortige Konfrontation mit der schwierigsten Situation) vorzuziehen. Ein massiertes Vorgehen birgt die Gefahr einer emotionalen Überforderung des Kindes. Expositionsübungen sollten einen möglichst hohen Bezug zu den realen Bedingungen haben. Daher ist eine Exposition in vivo (in der Realität) einer Exposition in sensu (in der Vorstellung) vorzuziehen. 
Eine ERM muss immer individuell auf den einzelnen Patienten und seine Zwänge ausgerichtet sein. Eine detaillierte Beschreibung des Vorgehens bei Expositionen für Kinder und Jugendliche mit Zwangsstörungen findet sich bei [35].

\section{HINTERGRUNDWISSEN}

Zentrale Punkte der Behandlungsstrategie: Exposition

- Einsicht in die Behandlungsstrategie (der Patient versteht und akzeptiert das Vorgehen)

- Erstellung einer Hierarchie von zwangsauslösenden Reizen (die Exposition beginnt graduell)

- Therapeutenbegleitung bei anfänglichen Expositionen

- häusliches Umfeld: Zwänge dort behandeln, wo sie auftreten

- Selbstmanagement: zunehmende Verantwortungsübernahme für die Exposition durch den Patienten

\section{Beispiele für erfolgreiche Behandlungskonzepte} basierend auf KVT

Im deutschsprachigen Raum liegt ein Therapiemanual für die Behandlung von Zwangsstörungen bei Kindern und Jugendlichen im Alter von 8-18 Jahren vor [35]. Dieses Manual ist für die Einzeltherapie konzipiert und für die ambulante Therapie zugeschnitten, aber auch bei stationären Patienten anwendbar. Es ist modular aufgebaut, die Module können flexibel kombiniert werden; damit ist die Dauer des Therapieprogramms variabel. Folgende Module finden sich:

- Modul I- Diagnostik,

- Modul II - Psychoedukation

- Modul III - erste therapeutische Eingrenzung

- Modul IV - kognitive Interventionen

- Modul V - Exposition mit Reaktionsmanagement

- Modul VI - Psychopharmakotherapie

- Modul VII - Nachsorge

\section{Merke}

Die Module Diagnostik, Psychoedukation und Nachsorge sollten grundsätzlich durchgeführt werden.

Jedes Modul hat einen ähnlichen Aufbau: Es ist gegliedert in Hintergrundwissen, Beschreibung der Sitzungen, Diskussion möglicher Probleme und enthält Fallbeispiele. Die Sitzungen sind im Ablauf gegliedert. Der Einstieg in die Sitzung beinhaltet gerade bei Kindern einen Elternkontakt. Daran schließt sich die Besprechung der therapeutischen Hausaufgabe an. Hierzu werden in einer Kinder- und Jugendlichenversion Info- und Arbeitsblätter eingesetzt.
Das Computerspiel Ricky und die Spinne (www.rickyandthespider.uzh.ch) soll die Chance auf eine möglichst frühzeitige verhaltenstherapeutische Behandlung kindlicher Zwangserkrankungen erhöhen und Psychotherapeuten in ihrer Arbeit mit zwangserkrankten Kindern unterstützen. In seinem Intro und den 8 „Levels“ sowie in Hausaufgaben integriert es die wichtigsten Behandlungselemente des verhaltenstherapeutischen Ansatzes. Ricky und die Spinne bietet eine ansprechende und kindgerechte Metapher, mit deren Hilfe die Zwangserkrankung, ihre Folgen und ihre Behandlung besser verstanden werden können.

Merke

Ein Computerspiel ermöglicht heute einen guten

Zugang zu Kindern, von denen dies meist als attraktiv empfunden wird.

Ricky und die Spinne kann nur im Rahmen einer Therapie eingesetzt werden. Eine erste Evaluation von Ricky und die Spinne an 18 zwangserkrankten Kindern zeigte, dass alle Kinder das Spiel schätzten und angaben, ihre Zwänge hätten sich durch die Behandlung stark gebessert. Die Therapeuten ihrerseits beurteilten Ricky und die Spinne als wertvolle Unterstützung bei der Behandlung von Zwangserkrankungen [38].

\section{Bedeutung der Angehörigen und Familieneinbindung}

Unter Familieneinbindung oder „Family Accommodation“ versteht man gut gemeintes, aber ungünstiges Verhalten von Eltern oder anderen Angehörigen, das zur Aufrechterhaltung einer Zwangserkrankung beiträgt und damit die Wirksamkeit einer verhaltenstherapeutischen Behandlung erschwert. Es geht dabei vor allem um eine übertriebene Anpassung des Familienalltags an die Zwänge, bis hin zur direkten Unterstützung bei deren Ausführung [39]. Bei kindlichen Zwangserkrankungen ist die Einbindung der Familie oft besonders ausgeprägt. Es kann zu sehr heftigen Szenen mit Beschimpfungen, aber auch körperlicher Gewalt seitens des Kindes kommen, wenn Eltern sich weigern, das Kind bei der Ausführung seiner Zwänge zu unterstützen [19]. Der Einbezug der Eltern in die verhaltenstherapeutische Behandlung eines zwangserkrankten Kindes ist deshalb längst allgemeiner Standard. In einer neueren Studie erwies sich das Ausmaß der Familieneinbindung als prognostisch relevanter Faktor für den Therapieerfolg [40]. Die gleiche Forschergruppe hat die Einbindung der Eltern bereits in der ersten Sitzung thematisiert und mit den Eltern gezielt alternatives Verhalten geübt [41]. Keine der Familien brach die intensive Behandlung ab, und die Eltern äußerten sich sehr zufrieden mit der Therapie. 


\section{Pharmakotherapie}

Die erste Wahl der Behandlung von Zwangsstörungen im Kindes- und Jugendalter ist wie beschrieben die KVT. In gewissen Fällen kann jedoch die Indikation für eine Pharmakotherapie gegeben sein. Eine weiterführende Darstellung der Pharmakotherapie von Kindern und Jugendlichen mit Zwangsstörungen findet sich im Lehrbuch Neuropsychopharmakotherapie des Kindes- und Jugendalters, Kapitel Behandlung der Zwangsstörung [42].

Die Kombination von KVT und Psychopharmakotherapie zeigte sich in der bisher größten kontrollierten Untersuchung (POTS 2004) am effektivsten [43]. In einer aktuellen Metaanalyse von 18 kontrollierten Studien zeigte sich für eine alleinige KVT eine Effektstärke von $d=1,203$, für eine alleinige pharmakologische Behandlung eine Effektstärke von $d=0,745$ und für eine kombinierte Behandlung von $d=1,704$ [44].

\section{Cave}

Eine Pharmakotherapie muss in jedem Fall in ein multimodales Behandlungsprogramm eingebettet sein.

\section{PRAXIS}

Indikation für eine Pharmakotherapie [42]

Die Indikation besteht, wenn die Symptome so ausgeprägt sind, dass das Kind oder der Jugendliche in seiner sozialen Integration (z. B. Schulbesuch nicht mehr möglich) massiv beeinträchtigt ist und eine KVT aufgrund der Schwere der Symptomatik nicht durchgeführt werden kann. In folgenden Situationen ist eine Pharmakotherapie angezeigt:

- Die Motivation für eine KVT ist (noch) nicht gegeben und die Bereitschaft dazu könnte durch die Medikation erhöht werden.

- Die KVT kann wegen bestehender Wartezeiten oder nicht vorhandener Ressourcen nicht durchgeführt werden.

- Es besteht eine deutlich ausgeprägte komorbide depressive Störung.

- Vorherrschende Zwangsgedanken.

\section{SSRI - Medikamente der ersten Wahl}

Selektive Serotonin-Wiederaufnahmehemmer (SSRI) sind die erste Wahl für die medikamentöse Behandlung einer Zwangsstörung im Kindes- und Jugendalter. SSRI zeigen ein gutes Wirkungsprofil bei vergleichsweise wenig Nebenwirkungen. Die Studienlage zeigt, dass die SSRI im Vergleich zu einem Placebo bei Kindern und Jugendlichen wirksam sind und zu einer klinisch relevanten Reduktion der Zwangssymptomatik führen. In kontrollierten Studien waren Fluoxetin, Fluvoxamin und Sertralin der Placebobehandlung überlegen [42]. In den meisten Studien wurde der Behandlungserfolg mit der schon erwähnten CY-BOCS ermittelt, die auch der Verlaufsmessung von Behandlungserfolg dient [34].

\section{Dosierungen von SSRI bei Zwangsstörungen im Kindes- und Jugendalter}

Da mit dem Wirkungseintritt von SSRI in der Regel erst nach 4-10 Wochen zu rechnen ist, ist auch ein möglicher Therapieerfolg erst nach 10-12 Behandlungswochen beurteilbar. Bei allen SSRI sollte die Tagesdosis am Morgen verabreicht werden. Einzig Fluvoxamin hat eine sehr viel kürzere Halbwertszeit und hier empfiehlt es sich, die Tagesdosis auf 2 Gaben, morgens und abends, aufzuteilen. In kontrollierten Untersuchungen wurden die SSRI höher dosiert, als es bei der Behandlung einer Depression im Kindes- und Jugendalter üblich ist. Die amerikanischen Leitlinien empfehlen ebenfalls eine regelmäßig höhere Dosierung, als in der Behandlung der Depression oder auch Angst üblich ist [45]. So werden Kinder und Jugendliche mit einer Depression überwiegend z. B. mit Fluoxetin mit einer Tagesdosis von 10-20 mg behandelt, während bei der Behandlung der Zwangsstörungen zwischen 20 und bis zu $60 \mathrm{mg}$ täglich eingesetzt wird.

\section{PRAXIS}

Vorgehensweise am Beispiel von Sertralin [42]

Bei Beginn der Therapie wird Kindern eine Tagesdosis von $25 \mathrm{mg}$ am Morgen verabreicht. Bei Jugendlichen kann auch mit $50 \mathrm{mg}$ begonnen werden. Dann wird wöchentlich um 25 mg bis zur Besserung der Zwangssymptomatik erhöht. Die Tageshöchstdosis liegt bei Kindern zwischen 50 und $100 \mathrm{mg}$ und bei Jugendlichen zwischen 75 und 200 mg.

\section{Medikation der zweiten Wahl}

Falls ein Patient nicht auf ein SSRI anspricht, sollte ein anderes SSRI gegeben werden - z. B. nach dem Beginn mit Sertralin dann Fluvoxamin. Neben SSRI wurde vor allem bei Erwachsenen das trizyklische Antidepressivum Clomipramin eingesetzt. In 2 Metaanalysen von randomisierten, kontrollierten Studien zu SSRI und Clomipramin bei pädiatrischen Patienten war Clomipramin noch wirksamer als die SSRI $[44,46]$. Es traten jedoch gerade im Kindes- und Jugendalter unter Clomipramin mehr unerwünschte Arzneimittelwirkungen und Studienabbrüche auf als unter den SSRI [47], daher wird es in der Regel nicht als „erstes“ Zweite-Wahl-Medikament eingesetzt.

Trizyklische Antidepressiva verfügen generell über eine geringe therapeutische Breite und weisen gegenüber SSRI ein erhöhtes Risiko für Intoxikationen und kardiologische Nebenwirkungen auf [48], [49]. Speziell bei Kindern und Jugendlichen sind SSRI demnach den trizyklischen Antidepressiva in der Behandlung von Zwangsstö- 
rungen, aber auch bei Angststörungen und Depression nach dem heutigen Wissensstand immer vorzuziehen.

PRAXIS

Zugelassene Medikation bei Zwangsstörungen im Kindes- und Jugendalter

In Deutschland sind Sertralin (ab dem 6. Lebensjahr) und Fluvoxamin (ab dem 8. Lebensjahr) für die Behandlung von Zwangsstörungen zugelassen.

Insgesamt sollte der Einsatz von Medikation aber immer zurückhaltend erfolgen. Langzeitwirkungen von SSRI auf das sich entwickelnde Gehirn sind kaum untersucht. Es gibt jedoch einige wenige Studien mit wiederum sehr kleinen Fallzahlen, die zeigen, dass Antidepressiva, und darunter auch die SSRI, Effekte auf die Mikrostruktur der weißen Substanz bei Patienten mit Zwangsstörungen haben könnten, die bei nicht medikamentös behandelten Patienten oder Gesunden nicht zu sehen waren [50].

SSRI können gerade im Jugendalter das Risiko für suizidale und aggressive Verhaltensweisen erhöhen [51]. Im Vergleich zum Erwachsenenalter kam es aber in den bisherigen Metaanalysen bei Kindern und Jugendlichen unter Studienbedingungen unter Medikation zu keinem Todesfall.

Merke

Unter Medikation sollte eine sehr engmaschige

Wirkungs- und Nebenwirkungskontrolle erfolgen.

\section{KERNAUSSAGEN}

Zwangsstörungen sind schon im Kindes- und Jugendalter häufig auftretende und sehr beeinträchtigende Erkrankungen. Die Erkrankung wird häufig lange verheimlicht oder bei Kindern nicht als solche erkannt. Wie bei nahezu allen psychischen Erkrankungen liegen häufig andere psychische Störungen komorbid vor. Hervorzuheben sind die Tic-Störungen, die - wenn sie gemeinsam mit Zwängen auftreten - eine besondere Entität zu bilden scheinen. Das Vorliegen oder Fehlen von Einsicht hat eine besondere diagnostische und therapeutische Relevanz. Die erste Wahl der Behandlung ist die kognitive Verhaltenstherapie. Sollte eine Kombination oder alleinige Psychopharmakotherapie indiziert sein, sind selektive Serotonin-Wiederaufnahmehemmer im Kindesund Jugendalter die erste Wahl. Der Einbezug der Eltern ist vor allem bei Kindern relevant. Je früher die Behandlung beginnt, umso besser ist die Prognose.
Interessenkonflikt

Susanne Walitza hat alle Interessenkonflikte öffentlich zugänglich deklariert unter https://www.uzh.ch/prof/ssl-dir/ interessenbindungen/client/web/

Über die Autoren

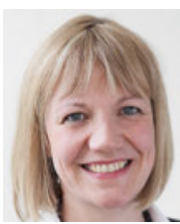

\section{Susanne Walitza}

Prof. Dr. med. Dipl.-Psych. Seit 2008 Direktorin der Klinik für Kinder- und Jugendpsychiatrie und -Psychotherapie der Psychiatrischen Universitätsklinik Zürich, Universität Zürich. Sie hat zum Thema Zwangsstörungen im Kindes-und Jugendalter habilitiert, leitet die Spezialsprechstunde für Tics und Zwänge und ist Vizepräsidentin der Schweizerischen Gesellschaft für Zwangsstörungen.

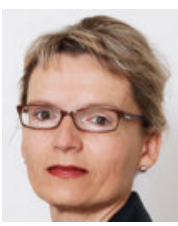

\section{Veronika Brezinka}

Dr. phil. Dr. (PhD). Psychologin und Verhaltenstherapeutin. Seit 2004 an der Klinik für Kinderund Jugendpsychiatrie und Psychotherapie der Psychiatrischen Universitätsklinik Zürich tätig. Seit 2009 ist sie Mitglied der Spezialsprechstunde für Tics und Zwänge. Sie ist Autorin der verhaltenstherapeutischen Computerspiele "Schatzsuche“ (www.treasurehunt.uzh.ch) und "Ricky und die Spinne“ (www.rickyandthespider.uzh.ch).

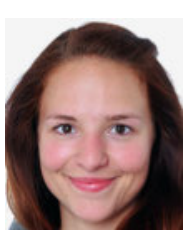

\section{Christa Rütter}

B. Sc. Masterstudentin der Klinischen Psychologie an der Universität Zürich. Sie war für die Fachstelle Autismus der Klinik für Kinder- und Jugendpsychiatrie und -Psychotherapie der Psychiatrischen Universitätsklinik Zürich tätig und hat dort in Forschungsprojekten und in der Vorbereitung der Leitlinien zum Thema Zwangsstörungen im Kindes- und Jugendalter mitgewirkt.

\section{Korrespondenzadresse}

Prof. Dr. med. Dipl.-Psych. Susanne Walitza

Klinik für Kinder- und Jugendpsychiatrie und -Psychotherapie Neumünsterallee 9

$\mathrm{CH}-8032$ Zürich

susanne.walitza@puk.zh.ch

Wissenschaftlich verantwortlich gemäß Zertifizierungsbestimmungen

Wissenschaftlich verantwortlich gemäß Zertifizierungsbestimmungen für diesen Beitrag ist Prof. Dr. med. Susanne Walitza, Zürich. 
[1] ICD-10-GM Version 2009: Internationale statistische Klassifikation der Krankheiten und verwandter Gesundheitsprobleme (Februar 2017). Im Internet: http://www.dimdi.de/static/de/ klassi/icd-10-gm/kodesuche/onlinefassungen/htmlgm2017/ block-f40-f48.htm/; Stand: 3.8.17

[2] American Psychiatric Association. Diagnostic and Statistical Manual of mental Disorders (DSM- $5^{\circledR}$ ). Arlington: American Psychiatric Association Publishing; 2013

[3] Walitza S. Zwangsstörungen im DSM-5. Was ist neu? Z Kinder Jugendpsychiatr Psychother 2014; 42: 121-127

[4] Schultze-Lutter F, Schimmelmann BG. Psychotische Störungen im DSM-5: die Revisionen. Z Kinder-Jugendpsychiatr Psychother 2014; 42: 193-202

[5] Lewin AB, Bergman RL, Peris TS et al. Correlates of insight among youth with obsessive-compulsive disorder. J Child Psychol Psychiatry 2010; 51: 603-611

[6] Geller DA, March J. Practice parameter for the assessment and treatment of children and adolescents with obsessivecompulsive disorder. J Am Acad Child Adolesc Psychiatry 2012; 51: 98-113

[7] Shimshoni Y, Reuven O, Dar R et al. Insight in obsessive-compulsive disorder: a comparative study of insight measures in an Israeli clinical sample. J Behav Ther Exp Psychiatry 2011; 42: 389-396

[8] Storch EA, Merlo L], Larson M] et al. Impact of comorbidity on cognitive-behavioral therapy response in pediatric obsessivecompulsive disorder. J Am Acad Child Adolesc Psychiatry 2008; 47: 583-592

[9] Garcia AM, Freeman JB, Himle MB et al. Phenomenology of early childhood onset obsessive compulsive disorder. J Psychopathol Behav Assess 2009; 31: 104-111

[10] Walitza S, Zellman H, Irblich B. Children and adolescents with obsessive-compulsive disorder and comorbid attention-deficit/hyperactivity disorder: preliminary results of a prospective follow-up study. J Neural Transm 2008; 115: 187-190

[11] Wewetzer C. Zwänge bei Kindern und Jugendlichen. Göttingen: Hogrefe; 2004

[12] Tagwerker Gloor F. Tic-Störungen im Kindes- und Jugendalter. PSYCH up2date 2015; 9: 161-176

[13] Walitza S, Melfsen S, Jans T et al. Obsessive-compulsive disorder in children and adolescents. Dtsch Arztebl Int 2011; 108: 173-179

[14] Storch EA, Lewin AB, Geffken GR et al. The role of comorbid disruptive behavior in the clinical expression of pediatric obsessive-compulsive disorder. Behav Res Ther 2010; 48: 12041210

[15] Leckman JF. Tourette's syndrome. Lancet 2002; 360: 15771586

[16] Kessler RC, Berglund P, Demler O et al. Lifetime prevalence and age-of-onset distributions of DSM-IV disorders in the National Comorbidity Survey Replication. Arch Gen Psychiatry 2005; 62: 593-602

[17] Delorme R, Golmard JL, Chabane N et al. Admixture analysis of age at onset in obsessive-compulsive disorder. Psychol Med 2005; 35: 237-243

[18] Stewart SE, Geller DA, Jenike M et al. Long-term outcome of pediatric obsessive-compulsive disorder: a meta-analysis and qualitative review of the literature. Acta Psychiatr Scand 2004; 110: 4-13
[19] Wewetzer C, Jans T, Müller B et al. Long-term outcome and prognosis of obsessive-compulsive disorder with onset in childhood or adolescence. Eur Child Adolesc Psychiatry 2001;10: 37-46

[20] Zellmann $\mathrm{H}$, Jans $\mathrm{T}$, Irblich B et al. Der mittelfristige Verlauf von Zwangsstörungen mit Beginn im Kindes- und Jugendalter: Aspekte der psychosozialen Anpassung. Verhaltensther Verhaltensmed 2008; 29: 336-351

[21] Zellmann $H$, Jans T, Irblich B et al. Kinder und Jugendliche mit Zwangsstörungen: Eine prospektive Verlaufsstudie. Z Kinder Jugendpsychiatr Psychother 2009; 37: 173-182

[22] Van Grootheest DS, Cath DC, Beekman AT et al. Twin studies on obsessive-compulsive disorder: a review. Twin Res Hum Genet 2005; 8: 450-458

[23] Steinhausen HC, Bisgaard C, Munk-Jørgensen P et al. Family aggregation and risk factors of obsessive-compulsive disorders in a nationwide three-generation study. Depress Anxiety 2013; 30: 1177-1184

[24] Walitza S, Wendland JR, Gruenblatt E et al. Genetics of earlyonset obsessive-compulsive disorder. Eur Child Adolesc Psychiatry 2010; 19: 227-235

[25] Van den Heuvel OA, van der Werf YD, Verhoef KM et al. Frontal-striatal abnormalities underlying behaviours in the compulsive-impulsive spectrum. J Neurol Sci 2010; 289: 55-59

[26] Brem S, Hauser TU, lannaccone R et al. Neuroimaging of cognitive brain function in paediatric obsessive compulsive disorder: a review of literature and preliminary meta-analysis. J Neural Transm (Vienna) 2012; 119: 1425-1448

[27] Menzies L, Chamberlain SR, Laird AR et al. Integrating evidence from neuroimaging and neuropsychological studies of obsessive-compulsive disorder: The orbitofronto-striatal model revisited. Neurosci Biobehav Rev 2008; 32: 525-549

[28] Snider LA, Lougee L, Slattery $M$ et al. Antibiotic prophylaxis with azithromycin or penicillin for childhood-onset neuropsychiatric disorders. Biol Psychiatry 2005; 57: 788-792

[29] Chang K, Frankovich ], Cooperstock M et al. Clinical evaluation of youth with pediatric acute-onset neuropsychiatric syndrome (PANS): recommendations from the 2013 PANS Consensus Conference. J Child Adolesc Psychopharmacol 2015; 25: 3-13

[30] Salkovskis PM. Understanding and treating obsessive-compulsive disorder. Behav Res Ther 1999; 37: 29-52

[31] Remschmidt H. Multiaxiales Klassifikationsschema für psychische Störungen des Kindes- und Jugendalters nach ICD-10 der WHO: mit einem synoptischen Vergleich von ICD-10 mit DSM-IV. Bern: Huber; 2006

[32] Schneider S. Kinder-DIPS: diagnostisches Interview bei psychischen Störungen im Kindes- und Jugendalter. Heidelberg: Springer; 2009

[33] Ambrosini PJ. Historical development and present status of the schedule for affective disorders and schizophrenia for school-age children (K-SADS). J Am Acad Child Adolesc Psychiatry 2000; 39: 49-58

[34] Scahill L, Riddle MA, McSwiggin-Hardin M et al. Children's Yale-Brown Obsessive-Compulsive Scale: reliability and validity. J Am Acad Child Adolesc Psychiatry 1997; 36: 844-852

[35] Wewetzer G, Wewetzer Ch. Zwangsstörung bei Kindern und Jugendlichen - Ein Therapiemanual. Göttingen: Hogrefe; 2012

[36] White M, Epston D. Narrative Means to therapeutic Ends. New York: Norton; 1990

[37] Lakatos A, Reinecker H. Kognitive Verhaltenstherapie bei Zwangsstörungen. 3. Aufl. Göttingen: Hogrefe; 2016 
[38] Brezinka V. Ricky and the Spider - a video game to support cognitive behavioural treatment of children with obsessivecompulsive disorder. Clin Neuropsychiatry 2013; 10 (Suppl. 1): $6-12$

[39] Renshaw KD, Steketee G, Chambless DL. Involving family members in the treatment of OCD. Cogn Behav Ther 2005; 34: $164-175$

[40] Rudy BM, Lewin AB, Geffken GR et al. Predictors of treatment response to intensive cognitive-behavioral therapy for pediatric obsessive-compulsive disorder. Psychiatry Res 2014; 220 : 433-440

[41] Lewin AB, Park JM, Crawford EA et al. Family-based exposure and response prevention therapy for preschool-aged children with obsessive-compulsive disorder: A pilot randomized controlled trial. Behav Res Therapy 2014; 56: 30-38

[42] Wewetzer C, Walitza S. Zwangsstörungen. In: Gerlach M, Mehler-Wex C, Walitza S, Warnke A, Wewetzer C, Hrsg. Neuro-Psychopharmaka im Kindes- und Jugendalter. 3. Aufl. Berlin: Springer; 2016: 611-618

[43] Pediatric OCD Treatment Study (POTS) Team. Cognitive-behavior therapy, sertraline, and their combination for children and adolescents with obsessive-compulsive disorder: the Pediatric OCD Treatment Study (POTS) randomized controlled trial. JAMA 2004; 292: 1969

[44] Sanchez-Meca J, Rosa-Alcazar A, Iniesta-Sepulveda M et al. Differential efficacy of cognitive-behavioral therapy and pharmacological treatments for pediatric obsessive-compulsive disorder: a meta-analysis. J Anxiety Disord 2014; 28: 31-44

[45] AACAP Official Action. Practice parameter for the assessment and treatment of children and adolescents. J Am Acad Child Psychiatry 2012; 51: 98-113
[46] Geller DA, Biedermann J, Stewart SE et al. Which SSRI? A metaanalysis of pharmacotherapy trials in pediatric obsessive-compulsive disorder. Am J Psychiatry 2003; 160: 1919-1929

[47] Gentile S. Efficacy of antidepressant medications in children and adolescents with obsessive-compulsive disorder. A systematic appraisal. J Clin Psychopharmacother 2011; 31: 625632

[48] Henry JA, Alexander CA, Sener EK. Relative mortality from overdose of antidepressants. Br Med J 1995; 310: 221-224

[49] Shah R, Uren Z, Baker A et al. Deaths from antidepressants in England and Wales 1993-1997: analysis of a new national database. Psychol Med 200; 31: 1203-1210

[50] Benedetti F, Giacosa C, Radaelli D et al. Widespread changes of white matter microstructure in obsessive-compulsive disorder: effect of drug status. Eur Neuropsychopharmacol 2013; 23: $581-593$

[51] Sharma T, Guski LS, Freund N et al. Suicidality and aggression during antidepressant treatment: systematic review and meta-analyses based on clinical study reports. $\mathrm{Br}$ Med J 2016; 352: i65

\section{Bibliografie}

DOI https://doi.org/10.1055/s-0043-103718

PSYCH up2date 2017; 11: 409-424

(c) Georg Thieme Verlag KG Stuttgart · New York ISSN 2194-8895 


\section{Punkte sammeln auf CME.thieme.de}

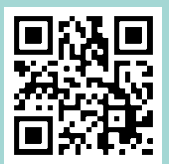

Diese Fortbildungseinheit ist 12 Monate online für die Teilnahme verfügbar.

Sollten Sie Fragen zur Online-Teilnahme haben, finden Sie unter cme.thieme.de/hilfe eine ausführliche Anleitung. Wir wünschen viel Erfolg beim Beantworten der Fragen!

Unter eref.thieme.de/ZZX8MXE oder über den QR-Code kommen Sie direkt zum Artikel zur Eingabe der Antworten.

VNR 2760512017152374569

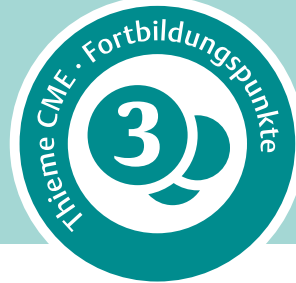

\section{Frage 1}

Wie hoch ist die Prävalenz von Zwangsstörungen im Kindesalter ungefähr?
A $0,5-1 \%$
B $1-3 \%$
C $5-10 \%$
D $20 \%$
E $25 \%$

\section{Frage 2}

Was ist typisch für Zwangsstörungen?

A Der Erkrankungsbeginn liegt im frühen Erwachsenenalter.

B Es besteht Einsicht in die Unsinnigkeit der Zwangsinhalte.

C Die Geschlechterverteilung ist ausgeglichen.

D Die Zwangssymptomatik führt zu einer Beeinträchtigung.

E Die Zwänge können auch angenehm sein wie ein Ritual.

\section{Frage 3}

Welche Aussage zur Komorbidität trifft zu?

A Zwänge haben in der Regel wenig Komorbidität.

$B$ Tic-Störungen sind bei Kindern und Jugendlichen am häufigsten.

C Autismus-Spektrum-Störungen treten besonders häufig auf.

D Es wird immer zuerst die Zwangsstörung behandelt.

E Bei Knaben tritt ADHS besonders häufig auf.

\section{Frage 4}

Welche Aussage trifft für die Unterscheidung von Tics und Zwängen zu?

A Zwanghaft wirkende Tics sind zweckgerichtet.

B Zwänge sind im Vergleich zu Tics oft zweckgerichtet.

C Tics und Zwänge können durch Beobachtung unterschieden werden.

D Tics werden ich-synton erlebt; Zwänge ich-dyston.

E Tics entwickeln sich früher in der Entwicklung als Zwangsstörungen.

\section{Frage 5}

Welche Aussage zu diagnostischen Verfahren von Zwangsstörungen im Kindes- und Jugendalter trifft zu?

A Da Zwänge bereits früh beginnen, wird die Diagnose meist vor der Pubertät gestellt.

B Die Grundlage für eine Diagnosestellung ist die Exploration und Beobachtung.

C Heute liegen zuverlässige neuropsychologische Testverfahren zur Diagnostik vor.

D Genetische Biomarker verbessern die klinische Diagnostik.

E Ein MRT muss zum Ausschluss einer Psychose vor der Diagnosestellung erfolgen.

\section{Frage 6}

Welche Aussage zu den Ursachen von Zwangsstörungen im Kindes- und Jugendalter trifft zu?

A Die Ursachen sind vor allem neurobiologische Faktoren der Hirnaktivität.

B Die Erziehung spielt die entscheidende Rolle.

C Mütterlicher Alkoholkonsum während der Schwangerschaft führt zu Zwangsstörungen beim Kind.

D PANDAS und PANS sind häufige Auslöser von Zwangsstörungen.

E Zwangsstörungen treten familiär gehäuft auf.

\section{Frage 7}

Welche Aussage zum Verlauf von Zwangsstörungen im Kindesund Jugendalter trifft zu?

A Der Verlauf bei Beginn im Kindesalter ist günstig.

B Je früher behandelt wird, umso besser ist der Verlauf.

C $30 \%$ der Erkrankten behalten ihre Zwangsstörung.

D Die Zwangsstörung wächst sich aus, während die Depression zunimmt.

E Remission und episodische Verläufe sind bei Beginn im Jugendalter häufig.

- Weitere Fragen auf der folgenden Seite... 


\section{Punkte sammeln auf CMIE.thieme.de}

Fortsetzung...

\section{Frage 8}

Welche Aussage zur KVT von Zwangsstörungen im Kindes- und Jugendalter trifft zu?

A Die KVT ist erst ab dem Jugendalter einzusetzen.

B Die zentrale Komponente bei der KVT ist die Elterneinbindung.

C Die Exposition wird im Kindesalter massiert eingesetzt.

D Wesentliches Element ist die Exposition mit Reaktionsverhinderung.

E Wirkt die KVT nicht, wird zusätzlich oder alleinig mit SSRI behandelt.

\section{Frage 9}

Welche Aussage zur Pharmakotherapie von Zwangsstörungen trifft zu?

A Die Therapie mit SSRI und Clomipramin ist die erste Wahl.

B Zwangsgedanken werden primär medikamentös behandelt.

C Das Intoxikationsrisiko von SSRI ist hoch.

D Als zweite Wahl werden Stimulanzien eingesetzt.

E Die Kombination von KVT und SSRI zeigt die höchste Effektivität.

\section{Frage 10}

Welche Aussage zur multimodalen Behandlung trifft zu?

A Die Diagnostik ist schon ein wesentlicher Behandlungsbaustein.

B Bei PANDAS hat sich eine Antibiotikaprophylaxe bewährt.

C Die Elterneinbindung spielt im Jugendalter keine Rolle mehr.

D Die kognitive Therapie spielt im Vergleich zur Exposition keine Rolle.

E Die Exposition sollte zu Beginn vor allem im stationären Setting stattfinden. 